\title{
Type III Polyketide Synthase $\beta$-Ketoacyl-ACP Starter Unit and Ethylmalonyl-CoA Extender Unit Selectivity Discovered by Streptomyces coelicolor Genome Mining
}

\author{
Lijiang Song $^{\dagger}$, Francisco Barona-Gomez ${ }^{\dagger}$, Christophe Corre ${ }^{\dagger}$, Longkuan Xiang ${ }^{\ddagger}$, Daniel W. \\ Udwary ${ }^{\ddagger}$, Michael B. Austin $\S$, Joseph P. Noel ${ }^{\S}$, Bradley S. Moore ${ }^{\ddagger}$, and Gregory L. Challis ${ }^{\star}, \dagger$ \\ tDepartment of Chemistry, University of Warwick, Coventry CV4 7AL, U.K. \\ ¥Scripps Institution of Oceanography, University of California, San Diego, California 92093 \\ §Salk Institute for Biological Studies, Howard Hughes Medical Institute, San Diego, California \\ 92186
}

\begin{abstract}
Polyketide synthases (PKSs) catalyze key carbon-carbon bondforming reactions in the biosynthesis of a diverse range of complex polyketide natural products, including many clinically useful antibacterial, anti-cancer, immunosuppressant, and anti-fungal compounds. 1 These reactions all involve the concomitant decarboxylation and condensation of a malonyl thioester with an acyl thioester and occur via a similar catalytic mechanism.1 PKSs have been classified into different types according to their sequence and primary structure, as well as the nature of the extender units they utilize in chain assembly.1 Type III PKSs occur commonly in plants, where they typically catalyze the iterative elongation of diverse acyl-CoA starter units with malonyl-CoA to form chalcone and stilbene products.2 Recently, genes encoding type III PKSs have been identified in bacterial genomes.2 These PKSs have been shown to typically catalyze the iterative decarboxylation and condensation of several malonyl-CoA molecules to form a poly- $\beta$-ketomethylene intermediate. This intermediate undergoes subsequent decarboxylation coupled with cyclization via Claisen and/or aldol reactions, followed by dehydration to give aromatic products such as $1,3,6,8-$ tetrahydroxynaphthalene, phloroglucinol, or 3,5-dihydroxyphenylacetyl coenzyme A, which is a precursor of the vancomycin non-proteinogenic amino acid residue 3,5dihydroxyphenylglycine.3,4 Analysis of the Streptomyces coelicolor A3(2) genome sequence has uncovered three open reading frames (ORFs) encoding potential type III PKSs, two of which are not associated with the production of known $S$. coelicolor metabolites.5 Here we report that one of these PKSs (Gcs), encoded by the sco $7221 \mathrm{ORF}$, is required for key reactions in germicidin biosynthesis.
\end{abstract}

The sequence between the start and stop codons of $s c 07221$ was replaced on the chromosome of $S$. coelicolor M145 by an 81 bp in-frame "scar" sequence using a recently developed PCR-targeting method.6 Cultures of the M145 strain and the $\Delta$ sco 7221 mutant were grown for 5 days in supplemented minimal medium (SMM). Comparative metabolic profiling of the culture supernatants of the wild type and the $\Delta$ sco 7221 mutant using LCESIMS identified two compounds with mass 196 and three compounds with mass 182 that were present in the wild type but lacking in the mutant (Figure 1). Complementation of the

(c) 2006 American Chemical Society

*G.L.Challis@warwick.ac.uk.

Supporting Information Available: Complete ref 5a, experimental procedures, and spectroscopic data. This material is available free of charge via the Internet at http://pubs.acs.org. 
$\Delta s c o 7221$ mutant with the plasmid pLS1 containing sco 7221 under the control of the $\mathrm{ermE}^{*}$ promoter restored production of these metabolites. High-resolution ESI-TOF-MS analysis gave $\mathrm{C}_{11} \mathrm{H}_{16} \mathrm{O}_{3}$ and $\mathrm{C}_{10} \mathrm{H}_{14} \mathrm{O}_{3}$ as the molecular formulas of the compounds of mass 196 and 182, respectively. A neutral loss of 44 mass units was observed in the negative-ion ESI-MS/ MS spectra, indicating the presence of a pyran-2-one in each of the compounds. The compounds were purified by semi-preparative HPLC from culture supernatants of $S$. coelicolor M145.1H, COSY, HMQC, HMBC, and NOE NMR analyses of the compounds of mass 196 identified them as germicidin A (1), previously isolated from Streptomyces viridochromogenes NRRL B-1551,7 and its novel isomer isogermicidin A (2). Similar analyses of the compounds with mass 182 indicated that they were germicidin B7 (3) and the new metabolites isogermicidin B (4) and germicidin C (5).

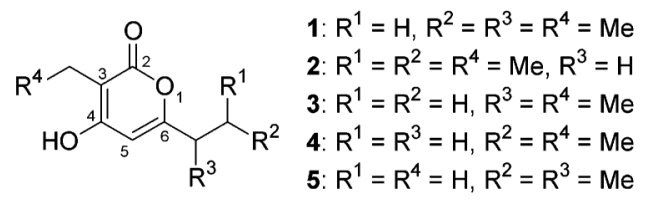

Inspection of the structures of $\mathbf{1 - 5}$ suggests that they may be assembled from 2methylbutyryl-CoA, isovaleryl-CoA, isobutyryl-CoA, or butyryl-CoA starter units by elongation with a malonyl-CoA or malonyl-ACP extender unit, followed by elongation with an ethylmalonyl-CoA or methylmalonyl-CoA extender unit. To examine the metabolic origin of the ethyl group attached to C-3 of $\mathbf{1 - 4}$, we fed $\left[{ }^{2} \mathrm{H}_{7}\right]$ butyric acid to cultures of $S$. coelicolor M145 grown in SMM. LC-ESIMS analyses of the culture supernatants revealed new compounds with the same retention time as $\mathbf{1}$ and $\mathbf{2}$ with $\mathrm{m} / z=201$ and 202, and new compounds with the same retention time as 3 with $\mathrm{m} / z=187$ and 188 , consistent with incorporation of four or five of the deuterium atoms from $\left[{ }^{2} \mathrm{H}_{7}\right]$-butyric acid into the ethyl groups attached to $\mathrm{C}-3$ of $\mathbf{1}, \mathbf{2}$, and $\mathbf{3}$. Similar analyses also revealed two new species with the same retention time as 4 with $\mathrm{m} / z=194$ and 195, consistent with the incorporation of four or five of the deuterium atoms from $\left[{ }^{2} \mathrm{H}_{7}\right]$-butyric acid into the $\mathrm{C}-3$ ethyl group and incorporation of all seven of the deuterium atoms of $\left[{ }^{2} \mathrm{H}_{7}\right]$ butyric acid into the C- $6 n$-propyl group of 4. These results indicate that butyric acid is incorporated intact into C-2, C-3, and the C-3 ethyl group of $\mathbf{1 - 4}$, consistent with the proposal that the second condensation reaction in the biosynthesis of these metabolites utilizes ethylmalonyl-CoA as the extender unit. They also indicate that butyric acid is incorporated intact into C- 6 and the $n$-propyl group attached to $\mathrm{C}-6$ of $\mathbf{4}$, consistent with the proposal that butyryl-CoA is the starter unit for biosynthesis of this compound. To investigate the role of 2-methylbutyryl-CoA as the starter unit for biosynthesis of $\mathbf{1}$, we fed $\left[\mathrm{U}^{13} \mathrm{C}\right]$ isoleucine to $S$. coelicolor M145. LCESIMS analysis of the culture supernatant identified a compound with the same retention time as 1 and $m / z=202$, consistent with intact incorporation of five contiguous carbon atoms of $\left[\mathrm{U}-{ }^{13} \mathrm{C}\right]$ isoleucine into $\mathrm{C}-6$ and the 2-methylpropyl group attached to $\mathrm{C}-6$ of $\mathbf{1}$, indicating that 2-methylbutyryl-CoA is the starter unit for biosynthesis of $\mathbf{1}$.

These results suggest two alternative models for the biosynthesis of 1-5 (Scheme 1). In the first model, the acyl-CoA starter unit is transacylated onto the active-site cysteine residue of Gcs, which subsequently catalyzes decarboxylation of malonyl-CoA and concomitant condensation with the starter unit to form the corresponding $\beta$-ketoacyl thioester $\mathbf{6}(\mathrm{X}=$ $\mathrm{SCoA}$ ). This thioester transacylates back onto the active-site cysteine residue of Gcs, which then catalyzes decarboxylation of ethyl- or methylmalonyl-CoA and concomitant condensation with the covalently bound $\beta$-ketothioester to give the corresponding $\beta, \delta$ diketothioester $\mathbf{7}(\mathrm{X}=\mathrm{SCoA})$. Finally, Gcs catalyzes the cyclization of $\mathbf{7}$ to give $\mathbf{1 - 5}$. In the second model, the $\beta, \delta$-diketothioester $7(\mathrm{X}=\mathrm{SCoA})$ is formed via transacylation of $\delta$ ketoacylthioester $6(\mathrm{X}=\mathrm{SACP})$ onto the activesite cysteine residue of Gcs. Decarboxylation 
of ethyl- or methylmalonyl-CoA and concomitant condensation with 6 gives 7, which undergoes the same cyclization reaction as in the first model to give the natural products. 6 $(\mathrm{X}=\mathrm{SACP})$ is a known intermediate in fatty acid biosynthesis in Streptomyces species that is formed by FabH- or FabF-catalyzed decarboxylation of malonyl-ACP and concomitant condensation with 2-methylbutyryl-, isovaleryl-, isobutyryl-CoA, or n-butyryl-ACP.8

To discriminate between these two models, we examined germicidin production in a recently reported strain of $S$. coelicolor M511 (a derivative of the M145 strain unable to produce actinorhodin) in which the $\mathrm{fabH}$ gene has been replaced by the orthologue from Escherichia coli.9 This strain produces predominantly straightchain fatty acids because the E. coli $\mathrm{FabH}$ is highly selective for acetyl-CoA as a starter unit.9 LC-ESIMS/MS analysis of culture supernatants from this strain grown in SMM showed that production of $\mathbf{1}, \mathbf{2}$, and $\mathbf{5}$ is abrogated, whereas small quantities ( $\sim 5 \%$ relative to wild type) of $\mathbf{3}$ and $\mathbf{4}$ still appear to be produced, consistent with the second model for germicidin biosynthesis presented above. To further examine the role of Gcs in germicidin biosynthesis, we transformed Streptomyces venezuelae ISP5230 with the plasmid pLS1. The complete genome sequence of $S$. venezuelae does not contain any orthologues of $s c o 7221$ (M. J. Bibb, personal communication), and LC-ESIMS analysis of culture supernatants of this organism grown in SMM indicated that it does not produce germicidins. LC-ESIMS/MS analysis of culture supernatants of $S$. venezualae/pLS1 grown in SMM showed that it produces 1-5, consistent with the hypothesis that only Gcs is required, in addition to enzymes of primary metabolism, for germicidin biosynthesis.

In conclusion, we have identified a novel bacterial type III PKS required for germicidin assembly that appears to catalyze elongation of specific $\beta$-ketoacyl-ACP thioester intermediates in fatty acid biosynthesis with ethyl- or methylmalonyl-CoA and subsequent cyclization of the resulting triketide. In vivo utilization of either ACP-tethered starter units or ethylmalonyl-CoA as an extender unit is unprecedented for any type III PKS.2 Modular type I PKSs are known to incorporate ethylmalonyl-CoA, 10 but only one other type III PKS has been proposed to utilize an extender unit other than malonyl CoA for the biosynthesis of a natural product. PstrCHS2, a plant type III PKS, is thought to incorporate methylmalonyl$\mathrm{CoA}$ in a modular fashion during the poorly understood biosynthesis of $\mathrm{C}$-methylated chalcones in the plant Pinus strobus.11

\section{Supplementary Material}

Refer to Web version on PubMed Central for supplementary material.

\section{Acknowledgments}

This work was supported by grants from the UK BBSRC and European Union (to G.L.C.) and the NIH (AI52443 to B.S.M. and J.P.N.). We thank Kevin Reynolds for providing S. coelicolor YL/ecFabH.

\section{References}

(1). Shen B. Curr. Opin. Chem. Biol. 2003; 7:285-295. [PubMed: 12714063]

(2). Austin MB, Noel JP. Nat. Prod. Rep. 2003; 20:79-110. [PubMed: 12636085]

(3)(a). Funa N, Ohnishi Y, Fujii I, Shibuya M, Ebizuka Y, Horinouchi S. Nature. 1999; 400:897-899. [PubMed: 10476972] (b) Zha W, Rubin-Pitel SB, Zhao H. J. Biol. Chem. 2006 DOI 10.1074/ jbc.M606500200.

(4)(a). Li T-L, Choroba O, Hong H, Williams D, Spencer J. Chem. Commun. 2001; 20:2156-2157.(b) Chen H, Tseng CC, Hubbard BK, Walsh CT. Proc. Natl. Acad. Sci. U.S.A. 2001; 98:1490114906. [PubMed: 11752437] 
(5)(a). Bentley SD, et al. Nature. 2002; 417:141-147. [PubMed: 12000953] (b) Austin MB, Izumikawa M, Bowman ME, Udwary DW, Ferrer J-L, Moore BS, Noel JP. J. Biol. Chem. 2004; 279:4516245174. [PubMed: 15265863]

(6). Gust B, Challis GL, Fowler K, Kieser T, Chater KF. Proc. Natl. Acad. Sci. U.S.A. 2003; 100:1541-1546. [PubMed: 12563033]

(7). Petersen F, Zahner H, Metzger JW, Freund S, Hummel RP. J. Antibiot. 1993; 46:1126-1138. [PubMed: 8360109]

(8). Han L, Lobo S, Reynolds KA. J. Bacteriol. 1998; 180:4481-4486. [PubMed: 9721286]

(9). Li Y, Florova G, Reynolds KA. J. Bacteriol. 2005; 187:3795-3799. [PubMed: 15901703]

(10)(a). Stassi DL, Kakavas SJ, Reynolds KA, Gunawardana G, Swanson S, Zeidner D, Jackson M, Liu H, Buko A, Katz L. Proc. Natl. Acad. Sci. U.S.A. 1998; 95:7305-7309. [PubMed: 9636144] (b) Hu Z, Reid R, Gramajo H. J. Antibiot. 2005; 58:625-633. [PubMed: 16392678]

(11). Schroder J, Raiber S, Berger T, Schmidt A, Schmidt J, Soares-Sello AM, Bardshiri E, Strack D, Simpson TJ, Veit M, Schroder G. Biochemistry. 1988; 37:8417-8425. [PubMed: 9622493] 


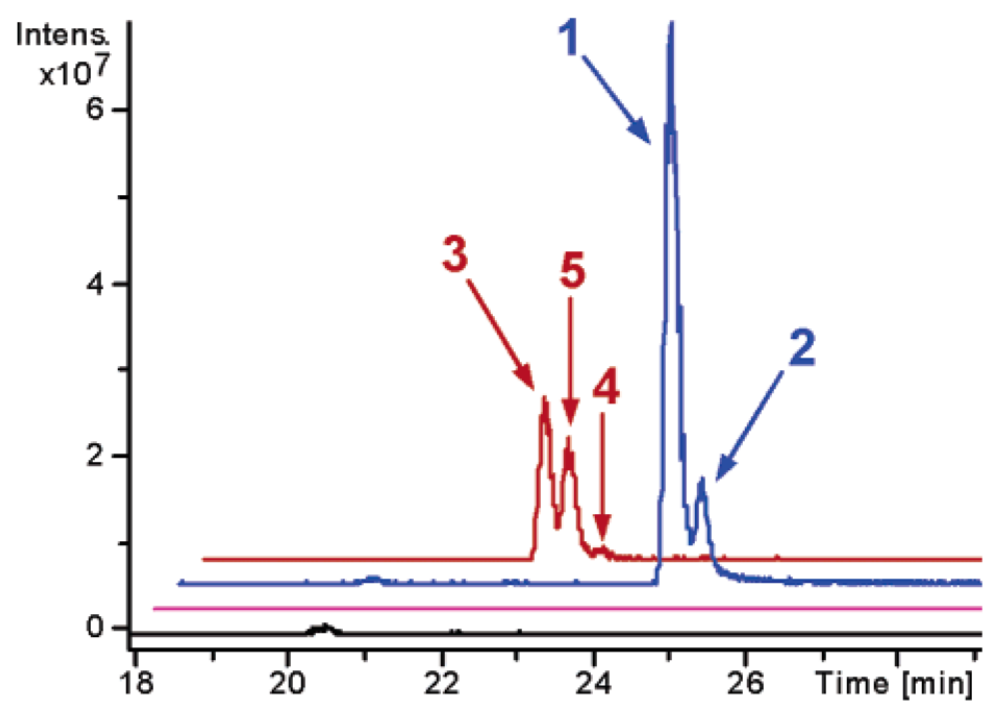

Figure 1.

LC-ESIMS extracted ion chromatograms at $\mathrm{m} / \mathrm{z}=197$ and 183 for organic extracts of $S$. coelicolor M145 (blue and red, respectively) and the sco7221::scar mutant (black and purple, respectively). 


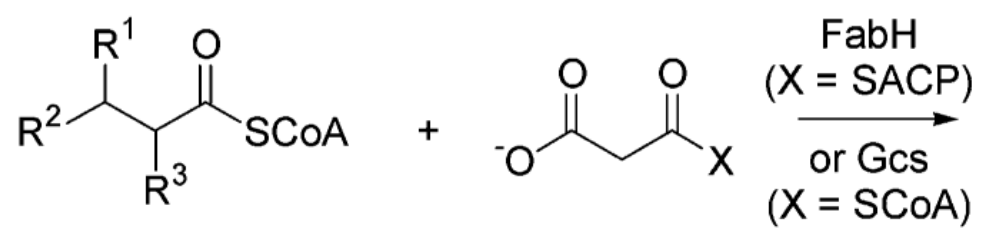

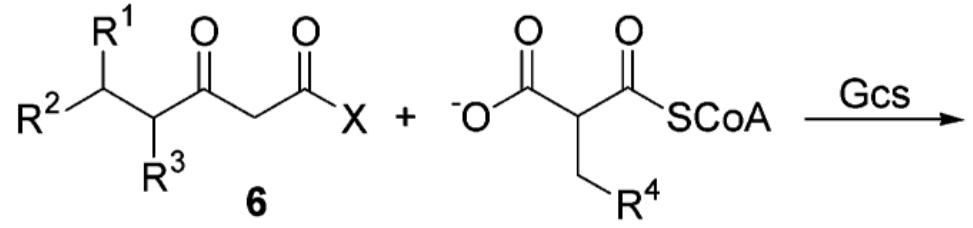

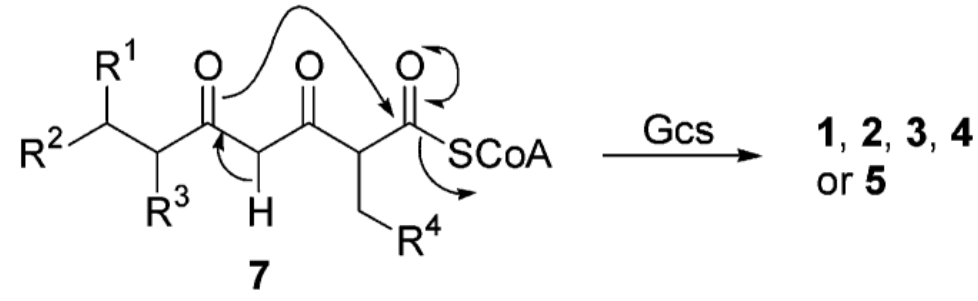

Scheme 1. 